\title{
Neural Correlates of Biased Competition in Premotor Cortex
}

\author{
Alexandre Pastor-Bernier and Paul Cisek \\ Département de Physiologie and Groupe de Recherche sur le Système Nerveux Central, Université de Montréal, Montréal, Québec H3C 3J7, Canada
}

It has been proposed that whenever an animal faces several action choices, their neural representations are processed in parallel in frontoparietal cortex and compete in a manner biased by any factor relevant to the decision. We tested this hypothesis by recording single-unit activity in dorsal premotor cortex (PMd) while a monkey performed two delayed center-out reaching tasks. In the one-target task, a single target was presented and its border style indicated its reward value. The two-target task was the same except two targets were presented and the value of each was varied. During the delay period of the one-target task, directionally tuned PMd activity showed no modulation with value. In contrast, during the two-target task, the same neurons showed strong effects of the value associated with their preferred target, always in relation to the value of the other target. Furthermore, the competition between action choices was strongest when targets were furthest apart. This angular distance effect appeared in neural activity as soon as cells became tuned, while modulation by relative value appeared much later. All of these findings can be reproduced by a computational model which suggests that decisions between actions are made through a biased competition taking place within a sensorimotor map of potential actions.

\section{Introduction}

Classical theories (Tversky and Kahneman, 1981) consider decision-making to be separate from the sensorimotor processes that implement the chosen response (Fodor, 1983). However, recent neurophysiological studies have shown neural correlates of decision variables within brain regions implicated in sensorimotor control (for review, see Glimcher, 2003; Gold and Shadlen, 2007; Cisek and Kalaska, 2010). For example, neural correlates of decision variables have been found throughout the saccade system, including the lateral intraparietal area (Platt and Glimcher, 1999; Dorris and Glimcher, 2004; Sugrue et al., 2004; Yang and Shadlen, 2007), the frontal eye fields (Schall and Bichot, 1998; Coe et al., 2002), and the superior colliculus (Basso and Wurtz, 1998; Horwitz et al., 2004), raising the question of why a putatively cognitive process should involve the sensorimotor system.

Such results appear less surprising if we consider that many of our everyday decisions are decisions between actions, such as choosing a path through a crowd or the target for a reach. It has been proposed that in such situations, the brain specifies several potential actions in parallel, and selects between them through a process of biased competition within the sensorimotor system itself (Cisek, 2007; Cisek and Kalaska, 2010). Recent computational models have suggested how multiple potential movements can be simultaneously encoded in parietal and premotor cortex (Tipper et al., 2000; Erlhagen and Schöner, 2002; Cisek, 2006;

Received Oct. 18, 2010; revised March 22, 2011; accepted March 24, 2011.

Author contributions: P.C. designed research; A.P.-B. performed research; A.P.-B. and P.C. analyzed data; A.P.-B. and P.C. wrote the paper.

This work was supported by research grants from the Canadian Institutes of Health Research and the ELB Foundation, a Groupe de Recherche sur le Système Nerveux Central doctoral fellowship to A.P-B., and an infrastructure grant from the Fonds de la Recherche en Santé du Québec. We thank Marie-Claude Labonté for technical support, and Pascal Poisson-Fortier and Trevor Drew for valuable comments regarding the manuscript and analyses.

Correspondence should be addressed to Dr. Paul Cisek, Département de physiologie, Université de Montréal, C.P. 6218 Succursale centre-ville, Montréal, QC, H3C 3J7, Canada. E-mail: paul.cisek@umontreal.ca.

DOI:10.1523/JNEUROSCI.5681-10.2011

Copyright $\odot 2011$ the authors $\quad 0270-6474 / 11 / 317083-06 \$ 15.00 / 0$
Furman and Wang, 2008), and how a competition between them can be biased by decision variables (Cisek, 2006).

This hypothesis makes several predictions. First, it predicts that neural activity can simultaneously represent several potential actions, as shown in the reaching (Cisek and Kalaska, 2005; Scherberger and Andersen, 2007) and grasping systems (Baumann et al., 2009), as well as in the saccade system (McPeek and Keller, 2002; Glimcher, 2003), where the influence of decision variables is already well established. Second, neural activity in sensorimotor regions will not represent any single decision variable in isolation, but will integrate all factors that influence choices. This implies that the variables associated with a given action will always be expressed relative to those associated with alternative actions. Third, the strength of competition between potential actions will depend on the similarity between them. This is motivated by simple facts of geometry: when choosing between two nearby targets, the nervous system can mix their neural representations and start moving between the targets. However, choosing between two targets in opposite directions implies that the choice has to be all-or-none. Here, we test these predictions through neural recordings in the dorsal premotor cortex (PMd) of a monkey performing a reach decision task, and compare the results to simulations of a biased competition model (Cisek, 2006). Some of these results have been presented previously in abstract form (Pastor-Bernier and Cisek, 2010).

\section{Materials and Methods}

A male monkey (Macaca mulatta) performed a planar center-out reaching task illustrated in Figure $1 \mathrm{~A}$ (see supplemental Methods, available at www.jneurosci.org as supplemental material). After a $350-650 \mathrm{~ms}$ center-hold-time (CHT), one or two cyan targets appeared, with border styles indicating their value in drops of juice (Fig. $1 A$, inset). The reward was determined probabilistically to encourage the monkey to explore available options (Herrnstein, 1961). A "low-value" target (L, thick border) had a $60 \%$ chance of yielding 1 drop, a $30 \%$ chance of yielding 2 drops, and a $10 \%$ chance of yielding 3 drops [expected value $(\mathrm{EV})=1.5$ ]. A "medium-value" target (M, no border) was worth 2 (60\%), 1 (20\%), or 
A

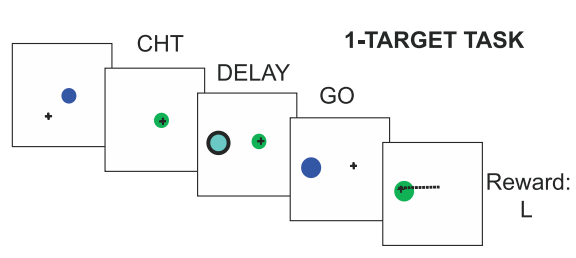

2-TARGET TASK

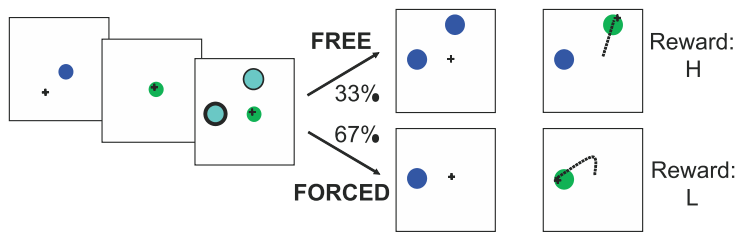

C
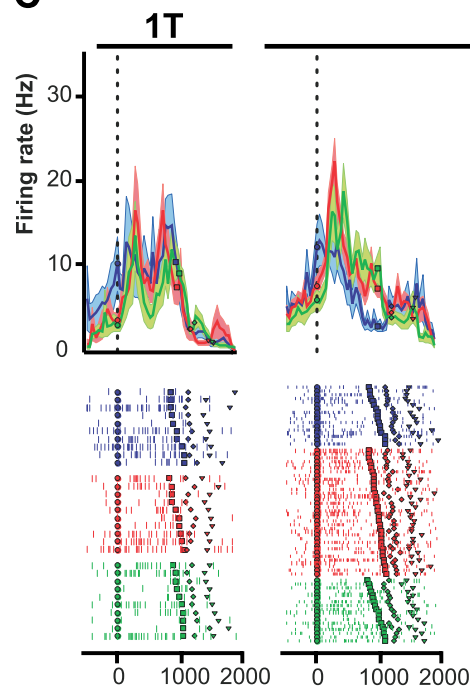

Time wrt Cue Onset (ms)
B
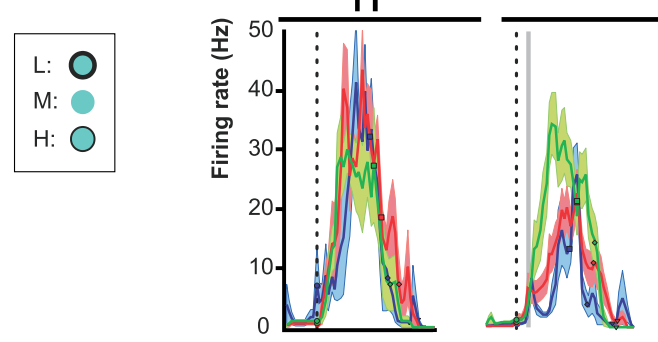

2T
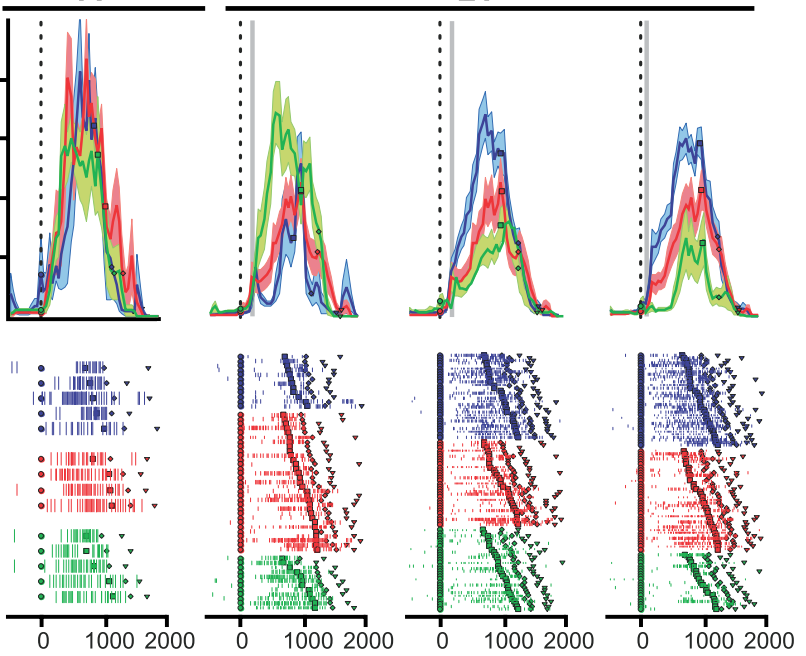

Time wrt Cue Onset (ms)
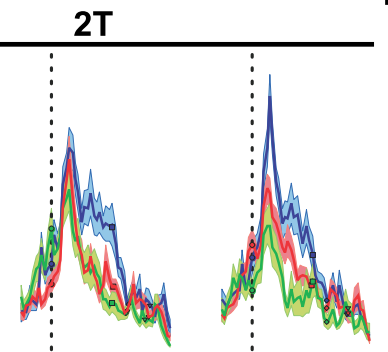

D

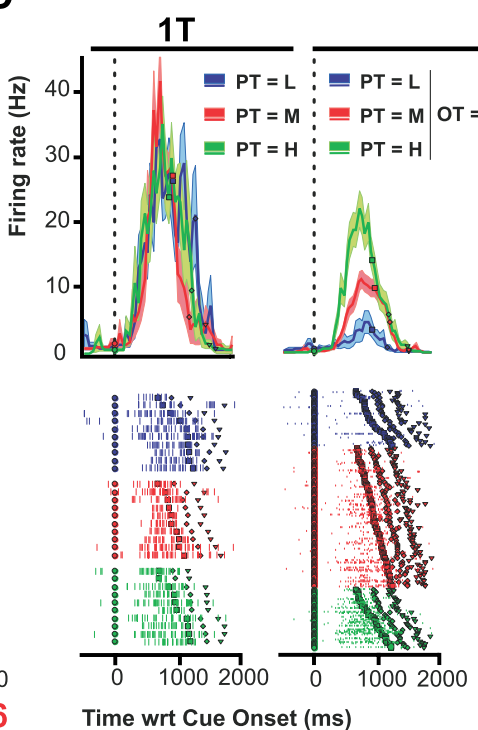

Time wrt Cue Onset (ms)
$2 T$
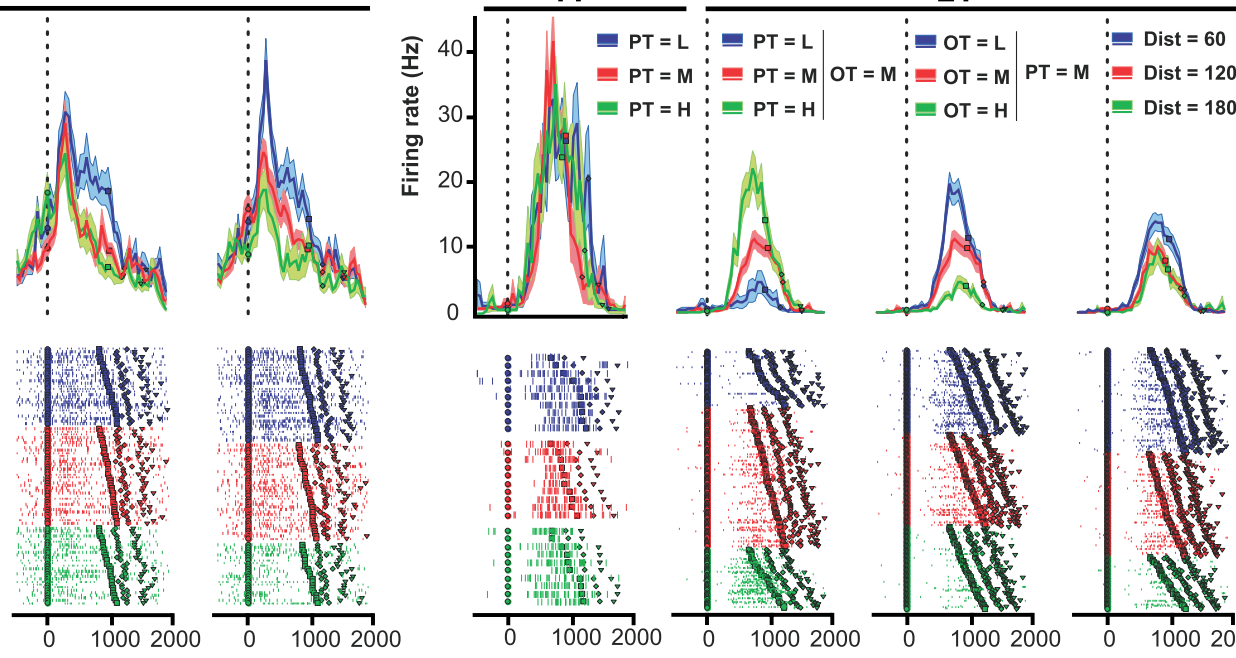

C46
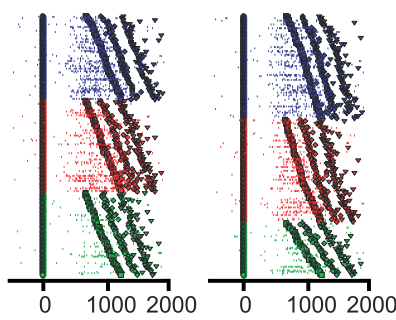

C189

Figure 1. A, Behavioral task. B-D, Three individual cell examples. Each panel shows histograms and raster plots for $1 \mathrm{~T}$ and $2 \mathrm{~T}$ trials in which the cell's PT was present. Activity is aligned on cue onset. The go signal, movement onset, and movement offset are indicated by thick squares, circles, and triangles, respectively. In the first column (1T task), colors indicate whether the PT value was low (blue), medium (red), or high (green). In the second column (2T) task, the PT values were low (blue), medium (red), or high (green), and there was also a medium-valued $0 T$ present. In the third column, the PT was always medium-valued while the $0 T$ value was low (blue), medium (red), or high (green). In the fourth column, both the PT and 0T were medium-valued, but the $0 T$ was $60^{\circ}$ (blue) $120^{\circ}$ (red), or $180^{\circ}$ (green) away from the PT. The vertical gray lines in $\boldsymbol{B}$ indicate the time when this cell's activity became statistically different $(p<0.01)$ in blue versus green conditions.

3 drops $(20 \%)(\mathrm{EV}=2)$. A "high-value" target $(\mathrm{H}$, thin border) was worth $3(60 \%), 2(30 \%)$, or 1 drop $(10 \%)(\mathrm{EV}=2.5)$. The nonmonotonic relationship between border thickness and value was used to dissociate motivational factors from physical properties of stimuli. The monkey held the cursor in the center for an instructed delay period (700-1300 ms) until a go signal was indicated by a change in target color and disappearance of the central circle. To receive the reward, the monkey had to move to a target within a maximum $550 \mathrm{~ms}$ movement time (MT) and hold the cursor there [target-hold-time (THT) $500 \mathrm{~ms}$ ].

When cells were isolated, we first ran a block of 90 trials in which only one target was presented (1T), to identify the delay-period preferred target (PT) of each cell. Next, we ran a block of 180 two-target trials (2T), including ones where the PT target was present and low-, medium-, or high-valued, while the other target (OT) appeared at $60^{\circ}, 120^{\circ}$, or $180^{\circ}$ away and was low-, medium-, or high-valued. Each block also included 30 trials in which the targets were $120^{\circ}$ apart, but neither was in the direction of the PT. These trials allowed us to analyze the activity of simultaneously recorded cells with different PTs. All analyses shown here use trials in which at least one of the targets presented was the cell's PT. In $33 \%$ of $2 \mathrm{~T}$ trials (free), the monkey was free to move to either target after the go signal. In $67 \%$ of $2 \mathrm{~T}$ trials (forced), one of the targets disappeared at go and the monkey had to move to the remaining target. Free and forced trials were randomly interleaved to encourage the animal to keep both options partially prepared.

To assess relative value effects, we compared delay-period activity during trials with targets $120^{\circ}$ apart in which the OT was medium-valued while the PT value varied ( $n \geq 60$ trials), as well as those in which the PT was mediumvalued while the OT value varied $(n \geq 60)$. To assess distance effects, we examined trials in which the PT was present and the OT was $60^{\circ}(n \geq 30)$, $120^{\circ}(n \geq 120)$ or $180^{\circ}$ away $(n \geq 30)$. Significance $(p<0.05)$ was assessed using two-tailed $t$ tests and ANOVA with post hoc Tukey-Kramer tests. Latency of effects was calculated as the time when the difference in activity between compared conditions exceeded 2 SDs in a sliding window (size, 10 $\mathrm{ms}$; step, $2 \mathrm{~ms}$ ) beginning at cue onset (Sato and Schall, 2003). 
A

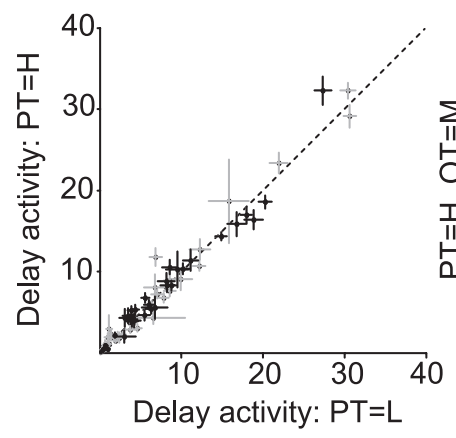

B

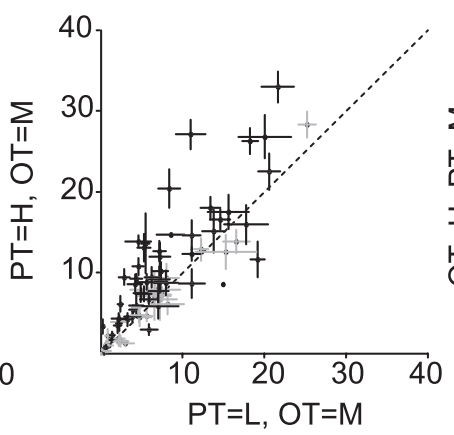

C

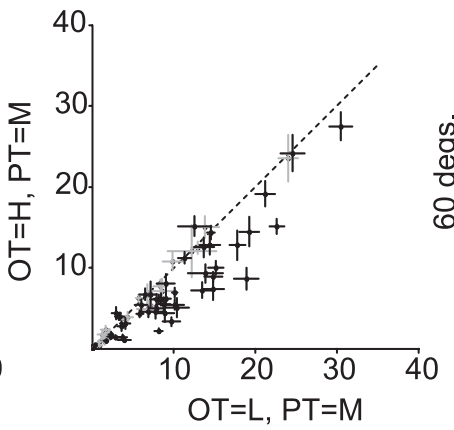

D

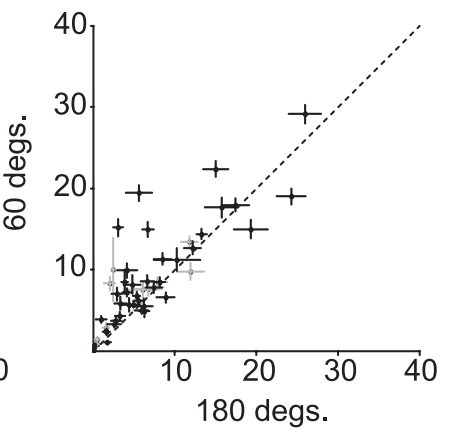

Figure 2. Population analyses. $\boldsymbol{A}$, Mean firing rate of individual cells in the $1 T$ task when the PT was low-valued ( $x$-axis) versus high-valued ( $y$-axis). Each cross indicates mean and SEM. $\boldsymbol{B}$, Firing rates comparing $2 \mathrm{~T}$ trials in which the $0 \mathrm{~T}$ is medium-valued and the PT is low-valued ( $x$ ) versus high-valued (y). C, Comparison of $2 \mathrm{~T}$ trials in which the PT is medium-valued and the $0 \mathrm{~T}$ is low-valued $(x)$ versus high-valued $(y) . D$, Comparison of $2 \mathrm{~T}$ trials in which the PT and OT are medium-valued and are $60^{\circ}(x)$ versus $180^{\circ}$ apart (y). In all panels, black crosses indicate cells with statistically significant effects ( $n=52$ ) along with the rest of the delay-tuned population ( $n=60$, gray).

A

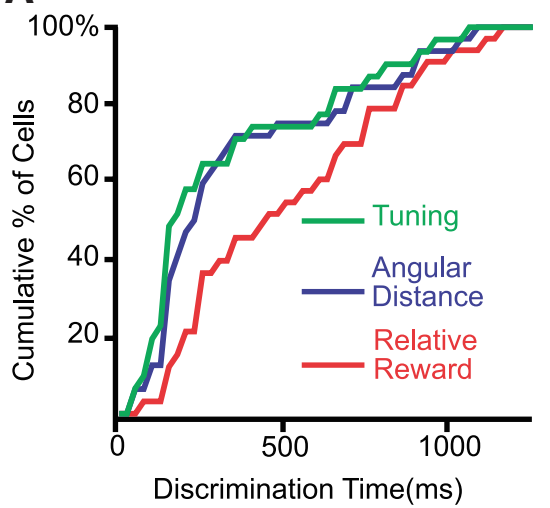

B

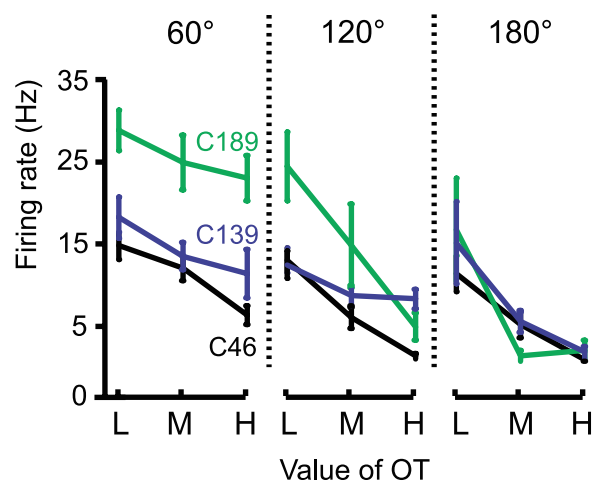

C

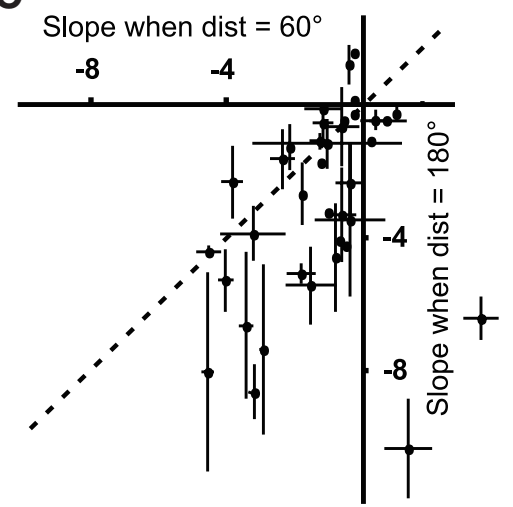

Figure 3. $A$, Cumulative distribution of latencies with which distance-complete cells ( $n=38$ ) exhibit tuning in the 1T task (green), and discriminate angular distance (blue) and relative value (red) in the 2 T task. $\boldsymbol{B}$, Firing rates of three example cells (Fig. $1 B-D$ ) as a function of OT value, when the PT was medium-valued. Each column shows trials with a different angular difference between targets $\left(60^{\circ}, 120^{\circ}, 180^{\circ}\right)$. Note that the slope is more negative for the $180^{\circ}$ trials. C, Comparison of the mean (and SEM) of the slopes in the $60^{\circ}$ versus $180^{\circ}$ conditions, for all distance-complete cells $(n=38)$.

To compare neural activity to model predictions (Cisek, 2006), we ran simulations of the same task and used similar analysis procedures. The model was identical to that previously described (Cisek, 2006), without any changes of parameters except that the model's "prefrontal" activity was scaled by a signal related to the absolute value of each target (low $=$ 0.3 , medium $=0.7$, high $=1.0$ )

\section{Results}

\section{Behavior}

In $1 \mathrm{~T}$ trials the monkey's success rate was $96 \%$, in $2 \mathrm{~T}$ free it was $96 \%$, and in $2 \mathrm{~T}$ forced it was $94 \%$ (in all cases $n>60,000$ ). In $2 \mathrm{~T}$ free trials the monkey selected the more valuable target $85 \%$ of the time, indicating that he understood the meaning of the stimulus cues.

Reaction times were similar across conditions because of the delay period. However, we observed a small but significant increase in movement speed to higher-valued targets: in the $1 \mathrm{~T}$ task, mean MT was $400 \mathrm{~ms}$ to high-value and $416 \mathrm{~ms}$ to low-value targets [Kolmogorov-Smirnov (KS) test, $p<0.01$ ].

\section{Neural activity in PMd}

Activity was recorded from 327 cells from the arm area of PMd (supplemental Fig. 1, available at www.jneurosci.org as supplemental material), of which 226 (69\%) had significant directional tuning during at least one epoch (delay, MT, THT) and were considered task-related. Here, we focus on cells with delay-period tuning ( 112 of $226,49 \%$ ). Approximately half of these (50 of 112, $45 \%$ ) were isolated long enough to collect data across all angular distances ("distance-complete" cells). Figure 1, $B-D$, shows the neural activity of three example cells, from trials in which each cell's PT was one of the targets presented. During the 1T task (first column), directionally tuned delay-period activity showed no effect of PT value. However, in the 2T task, when a second target was present and medium-valued (second column), the neural activity of all three cells now showed strong modulation with the relative value of the PT, firing more when their PT was more valuable than the OT (second column). This effect was also observed when the PT was medium-valued and the OT value was varied (third column). In this case, the cell activity was lower when the OT was more valuable than the PT. This finding suggests that the nature of the value effect is always relative to the other option presented.

Importantly, delay-period activity was also modulated as a function of the angular distance between the targets (Fig. $1 B-D$, fourth column). In most cases, activity was weaker when the targets were further apart $\left(180^{\circ}\right)$ than when they were closer to each other $\left(60^{\circ}\right.$ or $\left.120^{\circ}\right)$. Another interesting finding is the difference in latency between relative value and angular distance ef- 
A

$1 T$ task

Value effect
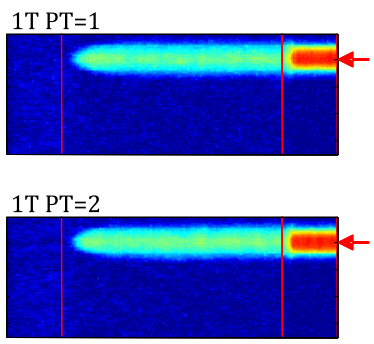

$1 \mathrm{~T} \mathrm{PT}=3$

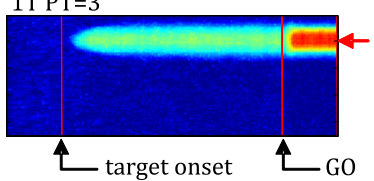

B

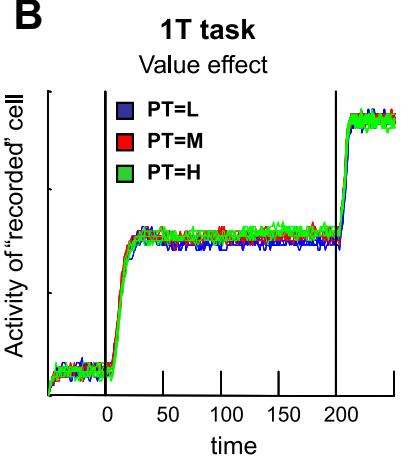

2T task

Effect of varying PT value
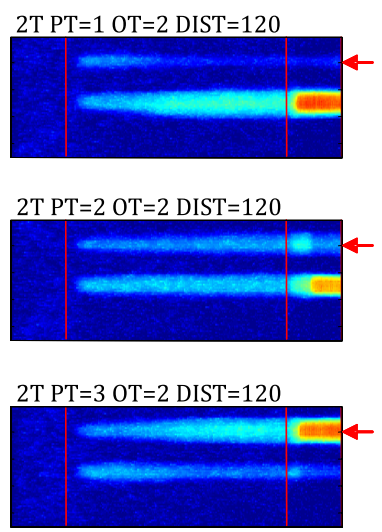

2T task

Effect of varying PT value

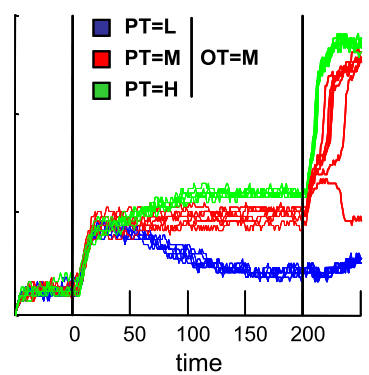

2T task

Effect of varying OT value
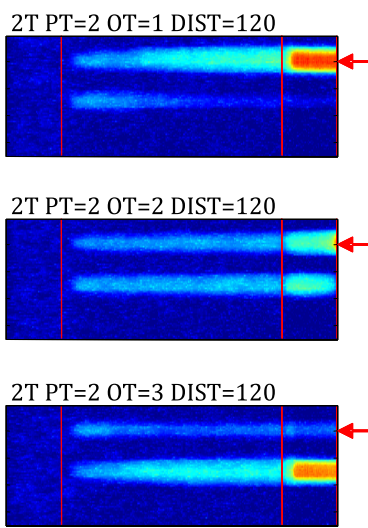

2T task

Effect of varying OT value

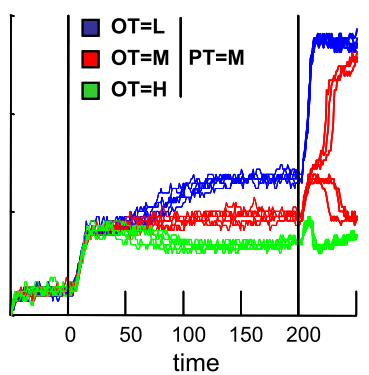

2T task

Effect of distance
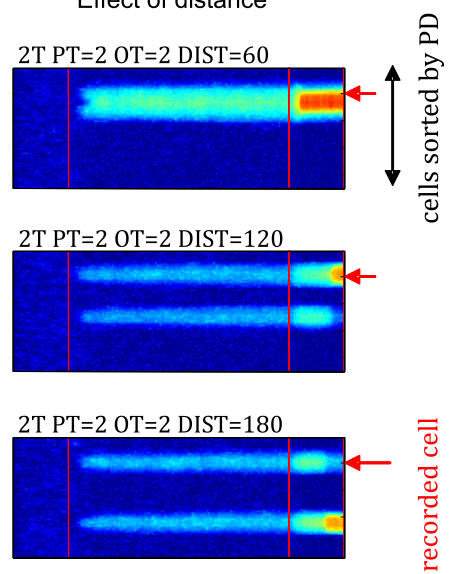

2T task

Effect of distance

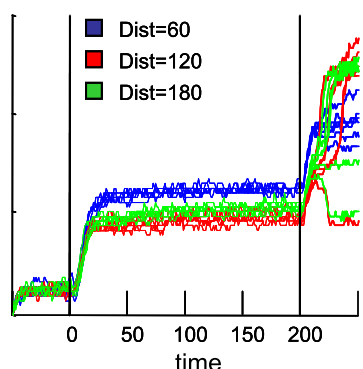

Figure 4. A, Activity from the model's caudal PMd population as color plots for 12 different conditions, as in the cell data. Each color plot shows activity evolving over the time course of a single trial ( $x$-axis), with cells sorted by their preferred direction ( $y$-axis). Blue indicates low activity and red indicates high activity. $B$, Behavior of one cell from the caudal PMd population, comparing activity across conditions as in the neural data (Fig. 1B-D).

fects. For example, the cell shown in Figure $1 B$ exhibited effects of angular distance $102 \mathrm{~ms}$ after target onset (fourth column), while the effects of expected value emerged significantly later, at $220 \mathrm{~ms}$ (third column).

\section{Population analyses}

The population of 112 delay-tuned cells was tested for relative value effects, and distance-complete cells were additionally tested for distance effects. From the entire tuned population of 112 cells, 49 (44\%) showed significant effects of relative value in the $2 \mathrm{~T}$ task $(t$ test, $p<0.05)$, with activity increasing with PT value and decreasing with OT value. Importantly, no effects were ever observed in the $1 \mathrm{~T}$ task ( $t$ test, $p>0.05$ for all comparisons). Across the group of distance-complete cells, 38 of 49 (78\%) showed some effect of relative value or distance. Thirty-five cells (71\%) showed relative value effects and $22(45 \%)$ showed angular distance effects (supplemental Table 1, available at www.jneurosci.org as supplemental material). Congruent results were obtained with $t$ tests and ANOVA with post hoc Tukey-Kramer tests ( $p<0.05$, see supplemental materials, available at www.jneurosci.org).

Figure $2 \mathrm{~A}$ compares the mean delay-period activity of individual cells $(n=112)$ during the 1T task when the PT was lowvalued ( $x$-axis) versus when it was high-valued ( $y$-axis). The means were not statistically different (Wilcoxon signed-rank test, $p=1)$. In contrast, most cells had higher delay activity in the $2 \mathrm{~T}$ task when the PT was more valuable than the OT (Fig. $2 \mathrm{~B}$, Wilcoxon signed-rank test, $p<10^{-6}$ ) and lower when the OT was worth more than the PT (Fig. $2 C, p<10^{-6}$ ). Approximately half (19 of $35,54 \%$ ) of the distance-complete cells with relative value effects also had stronger activity when the targets were $60^{\circ}$ apart than when they were $180^{\circ}$ apart (Fig. $1 D, p<10^{-3}$ ). Importantly, the same trends were observed across the entire population of cells with and without individually significant effects $(p>0.9$ in $1 \mathrm{~T}$; and $p<10^{-5}$ in $2 \mathrm{~T}$ for all comparisons). No significant effects of overall target value were found for cells that were not tuned during the delay $(p=1)$.

The latency of relative value and distance effects was calculated for all distance-complete cells with any effect $(n=38)$. Figure $3 \mathrm{~A}$ shows a cumulative distribution of the time at which a cell becomes tuned in the $1 \mathrm{~T}$ task, the time at which it exhibits a distance effect in the $2 \mathrm{~T}$ task, and the time at which it exhibits a relative value effect in the $2 \mathrm{~T}$ task. Across the population, effects of angular distance appeared at approximately the same time as cells became tuned, while the effect of relative value appeared $50-200 \mathrm{~ms}$ later. The relative-value and distance-effect distributions were statistically different (Kolmogorov-Smirnov test, $p<$ $0.024)$, as were the relative-value and tuning-onset distributions (KS test, $p<0.024)$. The difference between tuning-onset and distance-effect distributions was not statistically significant (KS test, $p>0.98$ ).

\section{Gain effect of distance over relative value}

Figure $3 B$ shows the mean delay-period activity of three example cells (Fig. $1 B-D$ ) as a function of OT value when the PT is 
medium-valued, separately for trials with targets $60^{\circ}, 120^{\circ}$, or $180^{\circ}$ apart. Note that all slopes are negative and steeper when targets are further apart. This suggests an interaction between angular separation and relative-value effects. Figure $3 C$ compares the slopes of all distance-complete cells with any effect $(n=38)$ when the targets are $60^{\circ}$ ( $x$-axis) versus $180^{\circ}$ ( $y$-axis) apart. The further apart the targets are, the more negative becomes the slope of activity versus relative value ( $t$ test, $p<0.003$ ).

\section{A biased competition model reproduces the results}

Cisek (2006) described a model of action selection in which populations of cells along the dorsal stream form a distributed representation of potential actions, which compete against each other through lateral inhibition (supplemental Fig. 2, available at www.jneurosci.org as supplemental material). The same model can simulate our neural recording results without any changes of parameters, except the addition of an absolute value signal into the prefrontal cortex (PFC) layer. As shown in Figure $4 A$, the model chooses the more valuable target when values are unequal and chooses randomly when they are equal. When targets are $60^{\circ}$ apart, the model often chooses the direction in-between the targets (Ghez et al., 1997). Figure $4 B$ shows an example of a simulated PMd neuron. Just as in real neurons, the simulated cell exhibits no sensitivity to value in the $1 \mathrm{~T}$ task. This is because the model continuously renormalizes activity across the population, and with one target it always produces one hill of activity that is similar regardless of biasing. However, the cell shows strong sensitivity to relative value in the $2 \mathrm{~T}$ task, in which the balance between two hills of activity can be influenced by biasing factors from PFC. The model also exhibits sensitivity to distance, with stronger activity when targets are $60^{\circ}$ apart than $120^{\circ}$ or $180^{\circ}$ apart. Finally, as in the data, the effect of distance is evident in the model almost immediately, but the effect of relative value takes longer to influence PMd activity because of the slow dynamics of model PFC (Fig. 4; note arbitrary time units).

\section{Discussion}

Recently, many studies have shown that decision variables influence neural activity throughout the sensorimotor system. These findings have sometimes been interpreted as the neural encoding of formal quantities such as uncertainty (Basso and Wurtz, 1998), expected gain (Platt and Glimcher, 1999), local income (Sugrue et al., 2004), or accumulated sensory evidence (Yang and Shadlen, 2007). We suggest that such findings do not necessarily imply that decision variables are explicitly encoded in neural activity (in the sense that they can be decoded), but may instead reflect their influence on a competition between potential actions taking place within the sensorimotor system. This predicts that any factor relevant for the monkey's choice will influence activity, including reward value, which was explicitly manipulated here. Importantly, however, our data show that the effect of value was always relative, and therefore never appeared when there was no choice to make. Our PMd results are therefore more naturally interpreted as motor-related activities that specify potential reach directions, which are modulated by relative subjective desirability (Dorris and Glimcher, 2004), a general term that includes all factors relevant to the choice.

While we found PMd activity to always reflect the relative values of actions, activity related to absolute values has been reported in the striatum (Samejima et al., 2005; Lau and Glimcher, 2008). It is possible that the basal ganglia are a major source of the biasing signal which influences premotor activity (Redgrave et al., 1999; Leblois et al., 2006; Cisek, 2007). In saccade tasks, activity related to absolute value has been reported in the parietal cortex (Platt and Glimcher, 1999; Seo et al., 2009) and in the ventral premotor cortex (PMv) (Roesch and Olson, 2003). The fact that we did not find reward-related modulations in PMd during our $1 \mathrm{~T}$ task may be attributable to differences between eye versus arm control or to differences in recording locations. For example, since PMv has response properties different from those of PMd (Boussaoud and Wise, 1993; Hoshi and Tanji, 2007), as well as distinct anatomical connections (Rizzolatti and Luppino, 2001), it may be more involved in representing sensory and reward information than PMd, which is more concerned with motor information. An earlier study using a saccade task (Roesch and Olson, 2004) found that PMd activity increased when either the reward or the penalty for one of the targets was increased. Although it is difficult to directly compare our results with those of a saccade task, in which PMd cells were not strongly directionally tuned, it is plausible that that effect was also related to relative subjective desirability.

One could argue that our findings are related to selective attention, which has also been described as biased competition (Desimone and Duncan, 1995). From the traditional perspective of cognitive psychology, one may wish to dissociate processes related to selective attention from those related to action selection. However, in our view (Cisek, 2007; Cisek and Kalaska, 2010), these may not be functionally distinct. It has been suggested that selective attention serves as an early mechanism for action selection (Allport, 1987; Neumann, 1990; Tipper et al., 1998), and that both are facets of the same biased competition occurring throughout the dorsal visuomotor stream (Duncan, 2006; Cisek, 2007). Indeed, it has been shown that microstimulation in a putatively motor region of frontal cortex can influence processing in visual cortex (Armstrong et al., 2006), demonstrating a strong link between attention and action selection.

Another important implication of our findings concerns the site of the competition that determines choices. Decision-related modulations in the sensorimotor system do not themselves necessarily imply that decisions are made within sensorimotor circuits. They could instead be made "upstream" in regions such as PFC, which are clearly involved in decisions (Tanji and Hoshi, 2001; Wallis and Miller, 2003) and project into sensorimotor regions. However, our results argue against this traditional view. First, we found that the dynamics of the competition that determines decisions are dependent on spatial variables. These are irrelevant for the abstract economics of cognition, but are important for the motor system, which selects between physical actions where geometrical relationships matter. Second, these effects of distance appear in cell activity as soon as cells respond to the stimuli, implying that the competition between potential actions takes place all throughout the fast sensorimotor "dorsal" visual stream (Cisek, 2007; Cisek and Kalaska, 2010). All of these results are remarkably well captured by a simple computational model (Cisek, 2006) which suggests the following conclusion: that although decisions between actions are influenced by variables supplied by higher cognitive regions, they are determined by a competition which takes place within sensorimotor circuits.

\section{References}

Allport DA (1987) Selection for action: some behavioral and neurophysiological considerations of attention and action. In: Perspectives on Perception and Action (Sanders HHAF, ed), pp 395-419. Hillsdale, NJ: Lawrence Erlbaum Associates.

Armstrong KM, Fitzgerald JK, Moore T (2006) Changes in visual receptive fields with microstimulation of frontal cortex. Neuron 50:791-798. 
Basso MA, Wurtz RH (1998) Modulation of neuronal activity in superior colliculus by changes in target probability. J Neurosci 18:7519-7534.

Baumann MA, Fluet MC, Scherberger H (2009) Context-specific grasp movement representation in the macaque anterior intraparietal area. J Neurosci 29:6436-6448.

Boussaoud D, Wise SP (1993) Primate frontal cortex: effects of stimulus and movement. Exp Brain Res 95:28-40.

Cisek P (2006) Integrated neural processes for defining potential actions and deciding between them: a computational model. J Neurosci 26:9761-9770.

Cisek P (2007) Cortical mechanisms of action selection: the affordance competition hypothesis. Philos Trans R Soc Lond B Biol Sci 362:1585-1599.

Cisek P, Kalaska JF (2005) Neural correlates of reaching decisions in dorsal premotor cortex: specification of multiple direction choices and final selection of action. Neuron 45:801-814.

Cisek P, Kalaska JF (2010) Neural mechanisms for interacting with a world full of action choices. Annu Rev Neurosci 33:269-298.

Coe B, Tomihara K, Matsuzawa M, Hikosaka O (2002) Visual and anticipatory bias in three cortical eye fields of the monkey during an adaptive decision-making task. J Neurosci 22:5081-5090.

Desimone R, Duncan J (1995) Neural mechanisms of selective visual attention. Annu Rev Neurosci 18:193-222.

Dorris MC, Glimcher PW (2004) Activity in posterior parietal cortex is correlated with the relative subjective desirability of action. Neuron 44:365-378.

Duncan J (2006) EPS Mid-Career Award 2004: brain mechanisms of attention. Q J Exp Psychol (Colchester) 59:2-27.

Erlhagen W, Schöner G (2002) Dynamic field theory of movement preparation. Psychol Rev 109:545-572.

Fodor JA (1983) The modularity of mind: an essay on faculty psychology. Cambridge, MA: MIT.

Furman M, Wang XJ (2008) Similarity effect and optimal control of multiple-choice decision making. Neuron 60:1153-1168.

Ghez C, Favilla M, Ghilardi MF, Gordon J, Bermejo R, Pullman S (1997) Discrete and continuous planning of hand movements and isometric force trajectories. Exp Brain Res 115:217-233.

Glimcher PW (2003) The neurobiology of visual-saccadic decision making. Annu Rev Neurosci 26:133-179.

Gold JI, Shadlen MN (2007) The neural basis of decision making. Annu Rev Neurosci 30:535-574.

Herrnstein RJ (1961) Relative and absolute strength of response as a function of frequency of reinforcement. J Exp Anal Behav 4:267-272.

Horwitz GD, Batista AP, Newsome WT (2004) Representation of an abstract perceptual decision in macaque superior colliculus. J Neurophysiol 91:2281-2296.

Hoshi E, Tanji J (2007) Distinctions between dorsal and ventral premotor areas: anatomical connectivity and functional properties. Curr Opin Neurobiol 17:234-242.

Lau B, Glimcher PW (2008) Value representations in the primate striatum during matching behavior. Neuron 58:451-463.
Leblois A, Boraud T, Meissner W, Bergman H, Hansel D (2006) Competition between feedback loops underlies normal and pathological dynamics in the basal ganglia. J Neurosci 26:3567-3583.

McPeek RM, Keller EL (2002) Superior colliculus activity related to concurrent processing of saccade goals in a visual search task. J Neurophysiol 87:1805-1815.

Neumann O (1990) Visual attention and action. In: Relationships between perception and action: current approaches (Prinz ONW, ed), pp 227-267. Berlin: Springer.

Pastor-Bernier A, Cisek P (2010) Neural correlates of biased competition between response options in dorsal premotor cortex. Abstract 1501, 7th FENS Forum of European Neuroscience, Amsterdam, the Netherlands, July 3-7, 2010.

Platt ML, Glimcher PW (1999) Neural correlates of decision variables in parietal cortex. Nature 400:233-238.

Redgrave P, Prescott TJ, Gurney K (1999) The basal ganglia: a vertebrate solution to the selection problem? Neuroscience 89:1009-1023.

Rizzolatti G, Luppino G (2001) The cortical motor system. Neuron 31: 889-901.

Roesch MR, Olson CR (2003) Impact of expected reward on neuronal activity in prefrontal cortex, frontal and supplementary eye fields and premotor cortex. J Neurophysiol 90:1766-1789.

Roesch MR, Olson CR (2004) Neuronal activity related to reward value and motivation in primate frontal cortex. Science 304:307-310.

Samejima K, Ueda Y, Doya K, Kimura M (2005) Representation of actionspecific reward values in the striatum. Science 310:1337-1340.

Sato TR, Schall JD (2003) Effects of stimulus-response compatibility on neural selection in frontal eye field. Neuron 38:637-648.

Schall JD, Bichot NP (1998) Neural correlates of visual and motor decision processes. Curr Opin Neurobiol 8:211-217.

Scherberger H, Andersen RA (2007) Target selection signals for arm reaching in the posterior parietal cortex. J Neurosci 27:2001-2012.

Seo H, Barraclough DJ, Lee D (2009) Lateral intraparietal cortex and reinforcement learning during a mixed-strategy game. J Neurosci 29:7278-7289.

Sugrue LP, Corrado GS, Newsome WT (2004) Matching behavior and the representation of value in the parietal cortex. Science 304:1782-1787.

Tanji J, Hoshi E (2001) Behavioral planning in the prefrontal cortex. Curr Opin Neurobiol 11:164-170.

Tipper SP, Howard LA, Houghton G (1998) Action-based mechanisms of attention. Philos Trans R Soc Lond B Biol Sci 353:1385-1393.

Tipper SP, Howard LA, Houghton, G (2000) Control of cognitive processes: attention and performance XVIII. Cambridge, MA: MIT.

Tversky A, Kahneman D (1981) The framing of decisions and the psychology of choice. Science 211:453-458.

Wallis JD, Miller EK (2003) From rule to response: neuronal processes in the premotor and prefrontal cortex. J Neurophysiol 90:1790-1806.

Yang T, Shadlen MN (2007) Probabilistic reasoning by neurons. Nature 447:1075-1080. 